\title{
On refusing resuscitation by ambulance staff
}

\author{
Raanan Gillon Imperial College, St Mary's Hospital Medical School and King's College, London University
}

In this issue of the journal Dr Kenneth Iserson raises the question of how patients can be enabled to refuse resuscitation by ambulance staff. Apparently in America ambulance staff are, in almost all cases, 'required to attempt resuscitation with all of the modalities at their disposal except in the most obvious cases of death' (1). While this is doubtless what a majority of patients would wish there are of course an important minority who certainly would not - among them some people with incurable and fatal diseases. Thus Dr Iserson describes a case which he says is typical of 'the multitude' - and illustrative of a 'common scenario in the United States'.

A woman with terminal metastatic pancreatic cancer was discharged from hospital having decided with her family and with the specialists and with the general practitioner to forego further treatment and die peacefully at home. However, when, about a week later, she lapsed into coma and into obvious respiratory distress her husband felt helpless and called the ambulance. The paramedic ambulance crew arrived, 'recognised the severity of the acute condition and began resuscitative procedures'. Despite the husband's protest that his wife was suffering from terminal cancer and did not want such interference the patient was fitted up with an endotracheal tube, an intravenous drip and a cardiac monitor, and was taken to the local hospital's casualty department where the intensive care was continued until her identity had been confirmed, her records obtained and her physician contacted - after which she was allowed to die. Fortunately the patient was not aware of her mode of dying, but her husband was, and in addition an advanced life-support ambulance and team were tied up for an hour as was the casualty intensive care team at the hospital, at a cost of about $\$ 1,300$. And had she been resuscitated to a state of awareness 'the moral tragedy would have been compounded by her additional pain and suffering accompanying the dying process for the second time' (1).

The first response to such a problem from most British and (other) European doctors and health care workers would doubtless be - 'thank goodness such an idiotic sequence of events could never happen here'. But why couldn't it happen in Europe? Several factors suggest themselves. Perhaps the most important, at least in Britain, is that common sense would prevent it. Common sense tells us all that it's daft to provide intensive care to someone whose husband has explained that his wife is terminally ill and does not want resuscitation. Yet the suspicious ambulanceman or woman may well ask: 'Why then did you telephone 999 (or 911 as it's more rapidly dialled in the USA)? How am I to be sure that what you tell me is true? For all I know you may have suddenly remembered that if she dies before she can rewrite her will you get all the money - whereas if she's resuscitated and rewrites it her new boyfriend - or the Old Horses Relief Fund will get the lot. How can I even be sure that you are her husband? Even if you are her husband and even if you are acting entirely honourably how can I be sure that you know what your wife is suffering from and that you know what her wishes are about resuscitation? In any case I am subject to strict protocols that require me to provide life-support techniques where a patient is apparently in danger of dying until or unless a doctor has ascertained that the patient is dead or that the patient has enacted a valid advance directive rejecting such life support interventions. Finally, I live and work in a culture whose members are very ready to sue each other in court and amongst whom there is a vocal minority who believe that life must be preserved wherever possible, regardless of the quality of that life. So common sense tells me, better safe than sorry.'

Such clashes of 'common sense' need further exploration. Underlying the American ambulance service position are several moral themes. The first is that the ideal determinant of action in such cases would be the patient's deliberated wishes - would the patient, having thought about the matter, want to be resuscitated or not? Here is a theme common to medical care on both sides of the Atlantic and perhaps everywhere. A second, more complex, moral theme is that where the health-care worker is in doubt about the patient's deliberated wishes it is better to presume in favour of saving life than otherwise. There are two components of this second theme, doubt and the presumption in favour of saving life. So far as doubt is concerned it is of course important to be reasonably sure of one's facts before taking any action affecting another's life, and the more potentially important the action the more important it is to try and be sure of 
one's facts. That said, it is never possible to be entirely certain about empirical facts - one must always act on a basis of probabilities, and with some probability that one's assessment of the facts will be wrong. The question then arises: which will be worse, to act or refrain from acting on the basis of what one reasonably believes to be the facts of the case or to act or desist from action on the basis that one might be wrong about the facts of the case? More specifically, in Dr Iserson's case history, which would be worse, for the ambulancemen to desist from resuscitation on the basis that they can reasonably believe that the man who called them is indeed the woman's husband, is indeed telling the truth, is indeed acting honourably, and does indeed know his wife's deliberated wishes in the matter; or to institute resuscitation against the express request of the husband on the grounds that they may be wrong in their reasonable belief that things are as the man has told them they are?

It seems quite clear that in such a case the probability that the ambulancemen would be going against the patient's wishes if they desisted from resuscitation would be extremely small. Conversely the probability that they would override the patient's wishes if they did institute resuscitation is clearly very high indeed. Thus in terms of respect for the patient's autonomous wishes, they would be far more likely to fulfil that moral obligation than not if they desisted from resuscitation than if they overrode the request of the 'putative' husband and resuscitated. Nor would this assessment alter if such a policy were generalised so that ambulancemen in general, while presuming that they ought to resuscitate people who were in apparent need of resuscitation nonetheless accepted instructions not to resuscitate from people purporting to be relatives or even merely friends of unconscious patients on the grounds that the patient would refuse such resuscitation. Again the issue seems to turn on the probabilities: such a policy may well lead to very rare cases where a patient's wishes to be resuscitated are overridden by the malice or ignorance of the friend or relative or other bystander who declines resuscitation. In the vast majority of cases, however, the patient's wishes are surely much more likely to be carried out if the ambulanceman accepts firm instructions to desist from resuscitation from those claiming to be friends or relatives and to know the patient's deliberated wishes than if ambulancemen resuscitate in all cases, even where such an instruction to the contrary is given. The probability of malicious or ignorant relatives wrongly rejecting resuscitation would be made even more minuscule if they were legally accountable for any such decision. Thus if the case were in any way suspicious, or if it were for any other reason to be investigated, the person declining resuscitation on behalf of another would have to be ready to justify his or her decision, in court if necessary. Such justification would require evidence of prior knowledge that the patient would have declined resuscitation; (or in the case of one who had never been competent to make such decisions, that the person declining resuscitation was the proper proxy and that it was not in the incompetent person's 'best interests' to be resuscitated).

So much for the doubt part of the moral theme: where in doubt about the patient's wishes it is better to presume in favour of saving life. What about the lifesaving part? This has always been the medical presumption and should surely continue to be so. The reason for this presumption is that people place enormous value on life, either as a precondition for the good life, or as a value in itself. But valuing life for either reason does not confer a general right on others to impose life-saving interventions upon a person against that person's deliberated choice to the contrary. Since by definition Dr Iserson's type of case involves patients who are unable to tell of their own deliberated choices about whether or not to accept such interventions the question once more collapses into the question considered above - which course of action is more likely to respect the patient's deliberated view about whether or not his or her life should be prolonged by resuscitation?

The theme that ambulancemen have to obey strict protocols that require them to resuscitate unless there is 'the most obvious case' of death, or unless a doctor instructs otherwise, merely begs the moral issue and indicates, if the foregoing analysis is correct, that such a strict protocol is mistaken. Here the British experience is instructive - a telephone inquiry made by the writer to a senior ambulanceman confirmed that there is no such strict protocol in British training for ambulance paramedics: 'It's left to the commonsense and judgement of the ambulanceman; people would normally be resuscitated but where the ambulanceman had reason to believe that someone was terminally ill he would be expected to allow that person to die with dignity'. As for the American tendency to go to court, that, like the question of making such decisions on the basis of scarcity of medical resources, requires separate assessment. Two points however are worth making. First, as Dr Iserson forcefully indicates, to insist on using intensive care resources on patients who deliberately reject them is clearly a waste of scarce medical resources. Second, courts too must confront the moral issues. If a central moral concern is not to impose medical care on patients who, having thought about the matter, wish to reject it, then the foregoing analysis is relevant in the courts too.

\section{References}

(1) Iserson $\mathrm{K}$ V. Foregoing prehospital care: should ambulance staff always resuscitate? Fournal of medical ethics 1991; 17: 19-24. 